UFIFT-QG-18-01

\title{
Structure Formation in Nonlocal MOND
}

\author{
L. Tan* and R. P. Woodard ${ }^{\dagger}$ \\ Department of Physics, University of Florida, \\ Gainesville, FL 32611, UNITED STATES
}

\begin{abstract}
We consider structure formation in a nonlocal, metric-based realization of Milgrom's MOdified Newtonian Dynamics (MOND). We derive the general equations for linearized scalar perturbations about the $\Lambda \mathrm{CDM}$ expansion history. These equations are considerably simplified for sub-horizon modes, and it becomes obvious (in this model) that the MOND enhancement is not sufficient to allow ordinary matter to drive structure formation. We discuss ways in which the model might be changed to correct the problem.
\end{abstract}

PACS numbers: 04.50.Kd, 95.35.+d, 98.62.-g

* e-mail: billy@ufl.edu

† e-mail: woodard@phys.ufl.edu 


\section{Introduction}

The failure of increasingly sensitive detectors to uncover any direct evidence for dark matter [1, 2] has motivated a re-examination of the dark matter paradigm. At the same time, powerful observational evidence [3, 4] continues to accumulate in support of the predictions of Milgrom's MOdified Newtonian Dynamics (MOND) [5, 6, 7] for galaxies. It seems that either MOND is correct or else the evolution of dark matter systems is driven towards a yet unrecognized attractor solution which reproduces the predictions of MOND.

MOND can be viewed as the nonrelativistic, static limit of some modified gravity theory in which cosmic motions are explained without the need for dark matter. Reconstructing this theory has proven to be challenging. The only local, stable, metric-based extensions of general relativity are $f(R)$ models [8], and these cannot reproduce MOND. We must therefore either abandon locality or give up the metric as the exclusive carrier of the gravitational force.

The first partially successful relativistic generalization of MOND was Bekenstein's TeVeS [9], which introduces scalar and vector fields to carry the extra gravitational force needed in the absence of dark matter. The creation of TeVeS quickly belied some of the facile pronouncements that had hitherto been made about the impossibility of an alternative to dark matter. For example, TeVeS does a better job of explaining large scale structure than had been thought possible [10, 11, 12, 13, 14]. The model does have some potential issues with stability [15], and suffers from the need for fine tuning to accommodate pulsar timing data [16]. More seriously, the model fails to agree with detailed measurements of lensing, clustering and structure growth [17, and it generically produces baryon acoustic oscillations which are too large [18]. The "gold-plated event" needed to completely falsify TeVeS was recently provided by the nearly simultaneous observation of gravitational wave and electromagnetic signals from a binary neutron star merger about $40 \mathrm{Mpc}$ away [19]. At this distance TeVeS predicts that gravitational waves should arrive hundreds of days before electromagnetic radiation [20].

Local relativistic generalizations of MOND which avoid the fatal problem of different arrival times employ other fields to change the gravitational field equations, but still use the metric to carry the gravitational force. A special type of Einstein-Aether models falls into this category [21, 22, 23]. A completely different class of models is provided by Milgrom's bimetric formulation [24, 25]. 
Fully metric-based generalizations of MOND cannot be local. Nonlocality is problematic in fundamental theory but it may be that MOND emerges from the nonlocal effective field equations which describe the gravitational vacuum polarization induced by primordial inflation [26]. This proposal is supported by the fact that loop corrections to the gravitational potentials during inflation show a small long range enhancement [27, 28, 29]. A purely phenomenological model was devised which reproduces the Tully-Fisher relation and sufficient weak lensing [30, 31]. When this model's free function was tuned to reproduce most of the $\Lambda$ CDM expansion history [32] a serendipitous explanation appeared for the tension between determinations of the current expansion rate derived from data at high [33] and low 34] redshift.

The purpose of this paper is to study cosmological perturbations. In section 2 we review the model. Section 3 presents the equations for linearized scalar perturbations around the cosmological background. At the end of section 3 we make the sub-horizon approximation and study the growth of structure. Section 4 discusses the implications for model-building.

\section{Past Work on the Model}

The purpose of this section is to review nonlocal MOND. We begin by giving the field equations for a general geometry. These equations are then specialized to static, spherically symmetric geometries, which reproduces the Tully-Fisher relation and weak lensing. Finally, the general equations are specialized to a homogeneous and isotropic background, which reproduces the $\Lambda$ CDM expansion history until a redshift of about $z_{*} \approx 0.088$.

\subsection{The Full Model}

Although the model is not local, its simplest formulation employs four auxiliary scalar fields to absorb the various nonlocal components after the technique of Nojiri and Odintsov [35. The localized Lagrangian depends upon the (spacelike) metric $g_{\mu \nu}$ and the auxiliary scalars $\phi, \xi, \chi$ and $\psi$ [31,

$$
\begin{aligned}
\mathcal{L}=\frac{c^{4}}{16 \pi G}\{R & +\frac{a_{0}^{2}}{c^{4}} f_{y}\left(\frac{g^{\mu \nu} \partial_{\mu} \phi \partial_{\nu} \phi}{c^{-4} a_{0}^{2}}\right) \\
& \left.-\left[\partial_{\mu} \xi \partial_{\nu} \phi g^{\mu \nu}+2 \xi R_{\mu \nu} u^{\mu} u^{\nu}\right]-\left[\partial_{\mu} \psi \partial_{\nu} \chi g^{\mu \nu}-\psi\right]\right\} \sqrt{-g}
\end{aligned}
$$


where $a_{0} \approx 1.2 \times 10^{-10} \mathrm{~m} / \mathrm{s}^{2}$ is the characteristic acceleration below which MOND phenomenology becomes apparent. The timelike 4-velocity field $u^{\mu}$ is formed from the gradient of $\chi$,

$$
u^{\mu} \equiv \frac{-g^{\mu \nu} \partial_{\nu} \chi[g]}{\sqrt{-g^{\alpha \beta} \partial_{\alpha} \chi[g] \partial_{\beta} \chi[g]}} .
$$

Regarding the four auxiliary scalars as independent fields would result in two ghosts [36, 37]. The scalars must instead be regarded as nonlocal functionals of the metric which are obtained by solving their field equations using retarded boundary conditions,

$$
\begin{gathered}
\phi[g]=\frac{2}{\square} R_{\alpha \beta} u^{\alpha} u^{\beta}, \chi[g]=-\frac{1}{\square} 1 \\
\xi[g]=\frac{2}{\square} D^{\mu}\left[\partial_{\mu} \phi f_{y}^{\prime}\left(\frac{g^{\rho \sigma} \partial_{\rho} \phi \partial_{\sigma} \phi}{c^{-4} a_{0}^{2}}\right)\right], \psi[g]=\frac{4}{\square} D_{\mu}\left[\frac{\xi\left(g^{\mu \rho}+u^{\mu} u^{\rho}\right) u^{\sigma} R_{\rho \sigma}}{\sqrt{-g^{\alpha \beta} \partial_{\alpha} \chi \partial_{\beta} \chi}}\right] .
\end{gathered}
$$

Here and henceforth $D_{\mu}$ denotes the metric-compatible covariant derivative. The full gravitational field equations are,

$$
\begin{aligned}
& R_{\mu \nu}+ \frac{1}{2} g_{\mu \nu}\left[-R-\frac{a_{0}^{2}}{c^{4}} f_{y}+g^{\rho \sigma}\left(\partial_{\rho} \xi \partial_{\sigma} \phi+\partial_{\rho} \psi \partial_{\sigma} \chi\right)+2 \xi u^{\rho} u^{\sigma} R_{\rho \sigma}-\psi\right] \\
&+\partial_{\mu} \phi \partial_{\nu} \phi f_{y}^{\prime}-\partial_{(\mu} \xi \partial_{\nu)} \phi-\partial_{(\mu} \psi \partial_{\nu)} \chi-2 \xi\left[2 u_{(\mu} u^{\alpha} R_{\nu) \alpha}+u_{\mu} u_{\nu} u^{\alpha} u^{\beta} R_{\alpha \beta}\right] \\
&-\left[\square\left(\xi u_{\mu} u_{\nu}\right)+g_{\mu \nu} D_{\alpha} D_{\beta}\left(\xi u^{\alpha} u^{\beta}\right)-2 D_{\alpha} D_{(\mu}\left(\xi u_{\nu)} u^{\alpha}\right)\right]=\frac{8 \pi G}{c^{4}} T_{\mu \nu} .
\end{aligned}
$$

The function $f_{y}(Z)$ which appears in equations (1), (41) and (5) is chosen to make the model agree with phenomenology in three regimes. The first is for small, positive $Z$, which corresponds to very weakly gravitationally bound systems whose Newtonian gravitational acceleration is comparable to $a_{0}$ or smaller. In this regime the Tully-Fisher relation implies [30],

$$
0<Z \ll 1 \quad \Longrightarrow \quad f_{y}(Z)=\frac{1}{2} Z-\frac{1}{6} Z^{\frac{3}{2}}+O\left(Z^{2}\right) \text {. }
$$

The second regime is for $Z \gtrsim 1$, which corresponds to more strongly gravitationally bound systems. Ensuring that general relativity applies in this regime requires $f_{y}(Z)$ to fall off rapidly. One function which meets this requirement, and is also consistent with ([6), is [31],

$$
0<Z<\infty \quad \Longrightarrow \quad f_{y}(Z)=\frac{1}{2} Z \exp \left[-\frac{1}{3} \sqrt{Z}\right] \text {. }
$$


The final regime is for negative $Z$, which corresponds to cosmology. In this regime the function was numerically fit to reproduce the $\Lambda$ CDM expansion history, without dark matter, from very early times until a redshift of $z_{*} \approx$ 0.088 32.

\subsection{Static, Spherically Symmetric Geometries}

The invariant element for static, spherically geometries can be expressed in terms of two potentials usually termed $A(r)$ and $B(r)$,

$$
g_{\mu \nu} d x^{\mu} x^{\nu}=-B(r) c^{2} d t^{2}+A(r) d r^{2}+r^{2} d \Omega^{2} .
$$

Specializing the general relations (2) and (3,4) to this geometry implies that the timelike 4-velocity field is,

$$
u^{\mu}=\frac{\delta_{0}^{\mu}}{\sqrt{B(r)}}
$$

The action of the scalar d'Alembertian on a function $F(r)$ is,

$$
\square F(r)=\frac{1}{r^{2} \sqrt{A B}} \frac{d}{d r}\left[r^{2} \sqrt{\frac{B(r)}{A(r)}} F^{\prime}(r)\right] .
$$

In the geometry (8) the 00 component and trace of the Ricci tensor are,

$$
\begin{aligned}
R_{00} & =\frac{B^{\prime \prime}}{2 A}-\frac{B^{\prime}}{4 A}\left(\frac{A^{\prime}}{A}+\frac{B^{\prime}}{B}\right)+\frac{B^{\prime}}{r A}, \\
R & =-\frac{B^{\prime \prime}}{A B}+\frac{B^{\prime}}{2 A B}\left(\frac{A^{\prime}}{A}+\frac{B^{\prime}}{B}\right)+\frac{2}{r A}\left(\frac{A^{\prime}}{A}-\frac{B^{\prime}}{B}\right)+\frac{2(A-1)}{r^{2} A} .
\end{aligned}
$$

Substituting expressions (9,11) into the general relations (3-4) gives $\psi=0$ and simple relations for the derivatives of $\phi(r)$ and $\xi(r)$,

$$
\phi^{\prime}(r)=\frac{B^{\prime}(r)}{B(r)} \quad, \quad \xi^{\prime}(r)=2 \phi^{\prime}(r) \times f_{y}^{\prime}\left(\frac{c^{4} B^{\prime 2}(r)}{a_{0}^{2} A(r) B^{2}(r)}\right) .
$$

From (13) we see that the argument of $f_{y}(Z)$ for this geometry is,

$$
Z=\frac{c^{4} B^{\prime 2}(r)}{a_{0}^{2} A(r) B^{2}(r)}
$$


We specialize the perfect fluid stress tensor,

$$
T_{\mu \nu}=(\rho+p) u_{\mu} u_{\nu}+p g_{\mu \nu},
$$

to this geometry with energy density $\rho(r)$ and pressure $p(r)$. Setting $\mu=$ $\nu=0$ in (5) gives,

$$
\frac{r A^{\prime}}{A^{2}}+1-\frac{1}{A}+\frac{a_{0}^{2}}{2 c^{4}} r^{2} f_{y}-\frac{r^{2} B^{\prime 2}}{A B^{2}} f_{y}^{\prime}-\frac{2}{\sqrt{A}} \partial_{r}\left[\frac{r^{2} B^{\prime}}{\sqrt{A} B} f_{y}^{\prime}\right]=\frac{8 \pi G}{c^{4}} r^{2} \rho .
$$

The $r r$ component of (5) implies,

$$
\frac{r B^{\prime}}{A B}-1+\frac{1}{A}-\frac{a_{0}^{2}}{2 c^{4}} r^{2} f_{y}+\frac{r^{2} B^{\prime 2}}{A B^{2}} f_{y}^{\prime}=\frac{8 \pi G}{c^{4}} r^{2} p .
$$

The other two nontrivial equations in this geometry are implied by stressenergy conservation.

If we assume the form (7) for $f_{y}(Z)$ equations (16, 17) can be reduced,

$$
\begin{array}{r}
\frac{8 \pi G}{c^{4}} r^{2} \rho=\left[r\left(1-\frac{1}{A}\right)\right]^{\prime}-\frac{r^{2} B^{\prime 2}}{4 A B^{2}}\left[1-\frac{c^{2} B^{\prime}}{3 a_{0} \sqrt{A} B}\right] \exp \left[-\frac{c^{2} B^{\prime}}{3 a_{0} \sqrt{A} B}\right] \\
-\frac{1}{\sqrt{A}} \partial_{r}\left\{\frac{r^{2} B^{\prime}}{\sqrt{A} B}\left[1-\frac{c^{2} B^{\prime}}{6 a_{0} \sqrt{A} B}\right] \exp \left[-\frac{c^{2} B^{\prime}}{3 a_{0} \sqrt{A} B}\right]\right\}, \\
\frac{8 \pi G}{c^{4}} r^{2} p=\frac{r B^{\prime}}{A B}-1+\frac{1}{A}+\frac{r^{2} B^{\prime 2}}{4 A B^{2}}\left[1-\frac{c^{2} B^{\prime}}{3 a_{0} \sqrt{A} B}\right] \exp \left[-\frac{c^{2} B^{\prime}}{3 a_{0} \sqrt{A} B}\right] .
\end{array}
$$

To understand how the Tully-Fisher relation and weak lensing emerge, set the pressure to zero and expand in the deviations,

$$
a(r) \equiv A(r)-1 \quad, \quad b(r) \equiv B(r)-1 .
$$

Note also that the length $\frac{c^{2}}{a_{0}} \simeq 7.5 \times 10^{26} \mathrm{~m}$ is greater than the current Hubble radius, so quadratic terms which lack this length can be discarded [38. Hence the equations become,

$$
\begin{aligned}
\frac{8 \pi G}{c^{4}} r^{2} \rho & \simeq\left[r a-r^{2} b^{\prime}+\frac{c^{2} r^{2} b^{\prime 2}}{2 a_{0}}\right]^{\prime} \\
0 & \simeq r b^{\prime}-a .
\end{aligned}
$$

Now use (22) - which gives lensing — to simplify (21) and integrate, recognizing the mass $M(r)$ enclosed within radius $r$,

$$
\frac{c^{2} r^{2} b^{\prime 2}}{2 a_{0}} \simeq \frac{2 G M(r)}{c^{2}} \Longrightarrow v_{\infty}^{2} \equiv \frac{1}{2} c^{2} \lim _{r \rightarrow \infty} r b^{\prime}(r)=\sqrt{a_{0} G M(\infty)} .
$$




\subsection{Homogeneous and Isotropic Geometries}

The invariant element for a spatially flat, homogeneous and isotropic geometry is,

$$
d s^{2}-c^{2} d t^{2}+a^{2}(t) d \vec{x} \cdot d \vec{x} \quad \Longrightarrow \quad H(t) \equiv \frac{\dot{a}}{a} .
$$

With initial time $t_{i}$, auxiliary scalars for this geometry are [31],

$$
\begin{array}{ll}
\phi_{0}(t)=6 \int_{t_{i}}^{t} \frac{d t^{\prime}}{a^{3}\left(t^{\prime}\right)} \int_{t_{i}}^{t^{\prime}} d t^{\prime \prime} a^{3}\left(t^{\prime \prime}\right)\left[H^{2}\left(t^{\prime \prime}\right)+\dot{H}\left(t^{\prime \prime}\right)\right] & \Longrightarrow Z_{0}(t)=-\frac{c^{2} \dot{\phi}^{2}(t)}{a_{0}^{2}}, \\
\chi_{0}(t)=\int_{t_{i}}^{t} \frac{d t^{\prime}}{a^{3}\left(t^{\prime}\right)} \int_{t_{i}}^{t^{\prime}} d t^{\prime \prime} a^{3}\left(t^{\prime \prime}\right) \quad \Longrightarrow \quad u_{0}^{\mu}(t)=\delta_{0}^{\mu}, \\
\xi_{0}(t)=2 \int_{t_{i}}^{t} d t^{\prime} \dot{\phi}_{0}\left(t^{\prime}\right) f_{y}^{\prime}\left(Z_{0}\left(t^{\prime}\right)\right) \quad, \quad \psi_{0}(t)=0 .
\end{array}
$$

We have bestowed a subscript 0 on these quantities to indicate that they represent the background solutions around which perturbations will be developed in the next section. The background gravitational field equations are [31],

$$
\begin{aligned}
3 H^{2}+\frac{a_{0}^{2}}{2 c^{2}} f_{y}\left(Z_{0}\right)+3 H \dot{\xi}_{0}+6 H^{2} \xi_{0} & =\frac{8 \pi G}{c^{2}} \rho_{0}, \\
-2 \dot{H}-3 H^{2}-\frac{a_{0}^{2}}{2 c^{2}} f_{y}\left(Z_{0}\right)-\ddot{\xi}_{0}-\left(\frac{\dot{\phi}_{0}}{2}+4 H\right) \dot{\xi}_{0}-\left(4 \dot{H}+6 H^{2}\right) \xi_{0} & =\frac{8 \pi G}{c^{2}} p_{0} .
\end{aligned}
$$

Here $\rho_{0}(t)$ and $p_{0}(t)$ are the background energy density and pressure without dark matter, which we parameterize using the cosmological redshift $z$,

$$
\begin{aligned}
\rho_{0}(t) & \equiv \frac{3 c^{2} H_{0}^{2}}{8 \pi G}\left[\Omega_{r}(1+z)^{4}+\Omega_{b}(1+z)^{3}+\Omega_{\Lambda}\right], \\
p_{0}(t) & \equiv \frac{3 c^{2} H_{0}^{2}}{8 \pi G}\left[\frac{1}{3} \Omega_{r}(1+z)^{4}-\Omega_{\Lambda}\right], \\
1+z & \equiv \frac{a\left(t_{0}\right)}{a(t)} .
\end{aligned}
$$

Whereas $\Omega_{r}$ and $\Omega_{\Lambda}$ are the $\Lambda$ CDM values for the fraction of critical density in radiation and vacuum energy, respectively, $\Omega_{b}$ is only the fraction of critical density in baryons. The fraction in nonrelativistic matter is $\Omega_{m}=\Omega_{c}+\Omega_{b}$, where $\Omega_{c} \approx 5.3 \times \Omega_{b}$ is the fraction in dark matter. 
For this cosmological regime the argument $Z$ of the function $f_{y}(Z)$ is negative. How the function $f_{y}(Z)$ depends on negative $Z$ is not fixed by MOND phenomenology. In a previous work [32] we showed how the function $f_{y}(Z)$ could be defined for $Z<0$ to exactly reproduce the $\Lambda$ CDM expansion, from very early times all the way up until a redshift of $z_{*} \approx 0.088$. The $\Lambda \mathrm{CDM}$ Hubble parameter rescaled by its current value $H_{0}$ is,

$$
\widetilde{H}(z)=\sqrt{\Omega_{r}(1+z)^{4}+\Omega_{m}(1+z)^{3}+\Omega_{\Lambda}} .
$$

The process of constructing $f_{y}(Z)$ to support $\widetilde{H}(z)$ without dark matter proceeds in two steps. We begin the first step by introducing the variables,

$$
f(z) \equiv-\frac{a_{0}^{2} f_{y}\left(Z_{0}\right)}{36 c^{2} H_{0}^{2} \Omega_{c}}, s(z) \equiv \frac{a_{0} \sqrt{-Z_{0}}}{6 c H_{0}}, g(z) \equiv \int_{z}^{\infty} \frac{f^{\prime}(\zeta) d \zeta}{(1+\zeta) s^{\prime}(\zeta) \widetilde{H}(\zeta)} .
$$

The first step consists of solving the following integral-differential equation for $f(z)$,

$$
\frac{1}{2} f(z)+\frac{\widetilde{H}(z) f^{\prime}(z)}{2 s^{\prime}(z)}+\widetilde{H}^{2}(z) g(z)=\frac{1}{12}(1+z)^{3} .
$$

In the second step we invert the relation for $s(z)$,

$$
s(z)=(1+z)^{3} \int_{z}^{\infty} d \zeta\left[\frac{(1+\zeta) \widetilde{H}^{\prime}(\zeta)-\widetilde{H}(\zeta)}{(1+\zeta)^{4}}\right] .
$$

to determine the redshift $z$ as a function of $Z_{0}$. Then regarding $f(z)$ as a function of $Z_{0}$ gives $f_{y}(Z)$ through relation (34). Although the function $s(z)$ is positive for large $z$, the presence of a cosmological constant causes it to vanish for $z=z_{*}$ [32], so we can only recover the $\Lambda$ CDM expansion history for $z_{*}<z<\infty$.

Relation (35) might seem to have two homogeneous solutions but it in fact has only one. To see this note first from expressions (33) and (36) that the large $z$ forms of $\widetilde{H}(z)$ and $s(z)$ are,

$$
\widetilde{H}(z) \longrightarrow \sqrt{\Omega_{r}}(1+z)^{2} \quad, \quad s(z) \longrightarrow \sqrt{\Omega_{r}}(1+z)^{2} .
$$

Now consider a homogeneous solution of (35) with an asymptotic large $z$ behavior of $(1+z)^{x}$. If we assume $x<4$ then the integral for $g(z)$ exists and we find an equation for the exponent $x$,

$$
f(z) \longrightarrow(1+z)^{x} \quad(x<4) \Longrightarrow \frac{1}{2}+\frac{1}{4} x+\frac{\frac{1}{2} x}{4-x}=0 \Longrightarrow x_{ \pm}=2(1 \pm \sqrt{3}) .
$$


The solution $x_{-}$is indeed less than 4 , so it corresponds to a genuine homogeneous solution. However, $x_{+}>4$, which means the derivation of this solution is not self-consistent. Hence there is only a single homogeneous solution, and equation (35) requires a single condition. Because the positive $Z$ function (6) vanishes for $Z=0$ it is natural to take this to be $f\left(z_{*}\right)=0$.
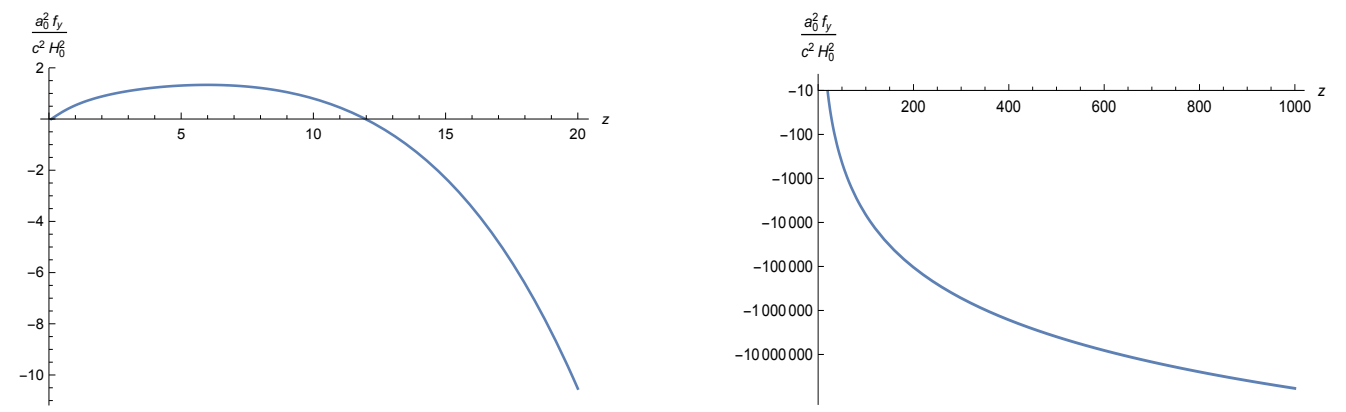

Figure 1: Both graphs show the quantity $\left(\frac{a_{0}}{c H_{0}}\right)^{2} \times f_{y}\left(Z_{0}\right)$ as a function of the redshift $z$. The left hand graph shows that $f_{y}\left(Z_{0}\right)$ vanishes at $z=z_{*} \approx 0.088$ and is positive for $z_{*}<z \lesssim 14$. The right hand graph shows that $f_{y}\left(Z_{0}\right)$ is negative, and monotonically decreasing, for all $z \gtrsim 12$.

A number of background quantities appear in the perturbation equations,

$$
\begin{aligned}
\frac{a_{0}^{2}}{c^{2} H_{0}^{2}} f_{y}\left(Z_{0}\right)=-36 \Omega_{c} \times f(z) \quad, \quad f_{y}^{\prime}\left(Z_{0}\right)=\frac{1}{2} \Omega_{c} \times \frac{f^{\prime}(z)}{s(z) s^{\prime}(z)}, \\
\xi_{0}(t)=-6 \Omega_{c} \times g(z) \quad, \quad \frac{\dot{\xi}_{0}(t)}{H_{0}}=-6 \Omega_{c} \times \frac{f^{\prime}(z)}{s^{\prime}(z)} \\
\frac{\dot{\phi}_{0}(t)}{H_{0}}=-6 \times s(z) \quad, \quad H_{0} \dot{\chi}_{0}(t)=(1+z)^{3} \int_{z}^{\infty} \frac{d \zeta}{(1+\zeta)^{4} \widetilde{H}(\zeta)} .
\end{aligned}
$$

It is important to know the signs, magnitudes and rough $z$ dependences of these quantities. Their behaviors for large $z$ follow from relation (37) for $s(z)$ and the implied relations $f(z) \longrightarrow \frac{1}{33}(1+z)^{3}$ and $g(z) \longrightarrow \frac{1}{22 \Omega_{r}} \frac{1}{1+z}$,

$$
\begin{aligned}
\frac{a_{0}^{2}}{c^{2} H_{0}^{2}} f_{y}\left(Z_{0}\right) \longrightarrow-\frac{12}{11} \Omega_{c}(1+z)^{3} \quad, \quad f_{y}^{\prime}\left(Z_{0}\right) \longrightarrow \frac{\Omega_{c}}{44 \Omega_{r}} \frac{1}{1+z}, \\
\xi_{0}(t) \longrightarrow-\frac{3 \Omega_{c}}{11 \Omega_{r}} \frac{1}{1+z} \quad, \quad \frac{\dot{\xi}_{0}(t)}{H_{0}} \longrightarrow-\frac{3 \Omega_{c}}{11 \sqrt{\Omega_{r}}}(1+z), \\
\frac{\dot{\phi}_{0}(t)}{H_{0}} \longrightarrow-6 \sqrt{\Omega_{r}}(1+z)^{2} \quad, \quad H_{0} \dot{\chi}_{0}(t) \longrightarrow \frac{1}{5 \sqrt{\Omega_{r}}} \frac{1}{(1+z)^{2}} .
\end{aligned}
$$



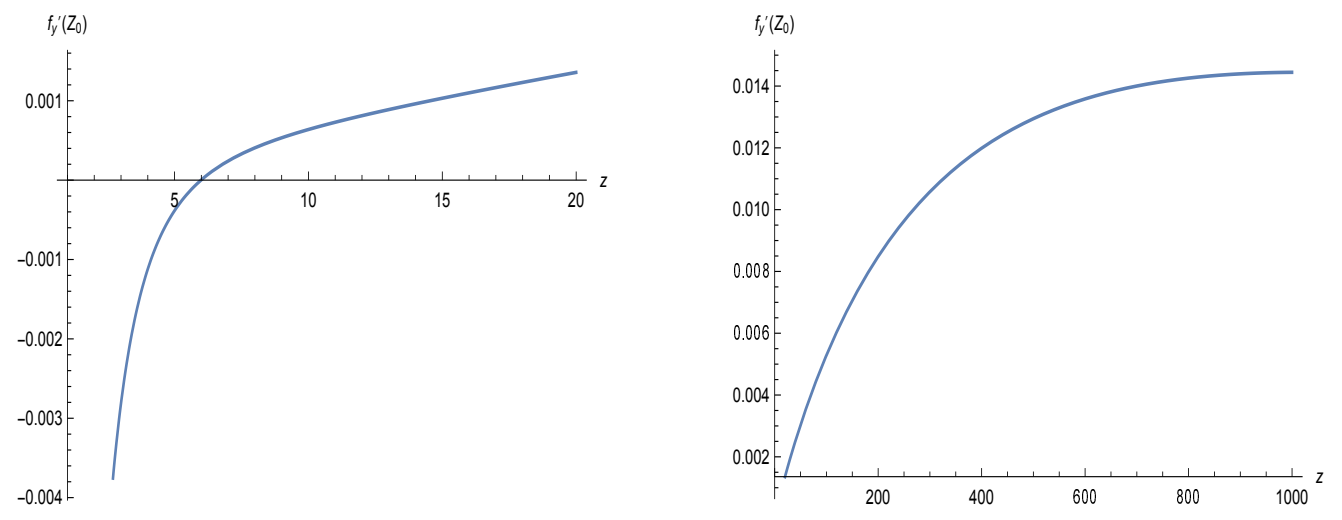

Figure 2: Both graphs depict the quantity $f_{y}^{\prime}\left(Z_{0}\right)$ as a function of the redshift $z$. The left hand graph shows that $f_{y}^{\prime}\left(Z_{0}\right)$ is negative for $z \lesssim 6$, and approaches $-\infty$ at $z=z_{*} \approx 0.088$. The right hand graph demonstrates that $f_{y}^{\prime}\left(Z_{0}\right)$ never becomes larger than about 0.015 .

Although the large $z$ results (42,44) are valid, there is no alternative to numerically evaluating the six quantities (39-41) for the redshifts of relevance to structure formation. Fig. 1 shows the function $f_{y}\left(Z_{0}\right)$ - rescaled by $\left(\frac{a_{0}}{c H_{0}}\right)^{2}$ - as a function of the redshift $z$. Fig. 2 depicts $f_{y}^{\prime}\left(Z_{0}\right)$ versus $z$, demonstrating both that the function approaches $-\infty$ at $z=z_{*}$, and that it never becomes more positive than about 0.015 in the range of interest for structure formation. That turns out to be the crucial point in dooming this model. Figures 3 and 4 show the other relevant auxiliary scalars.
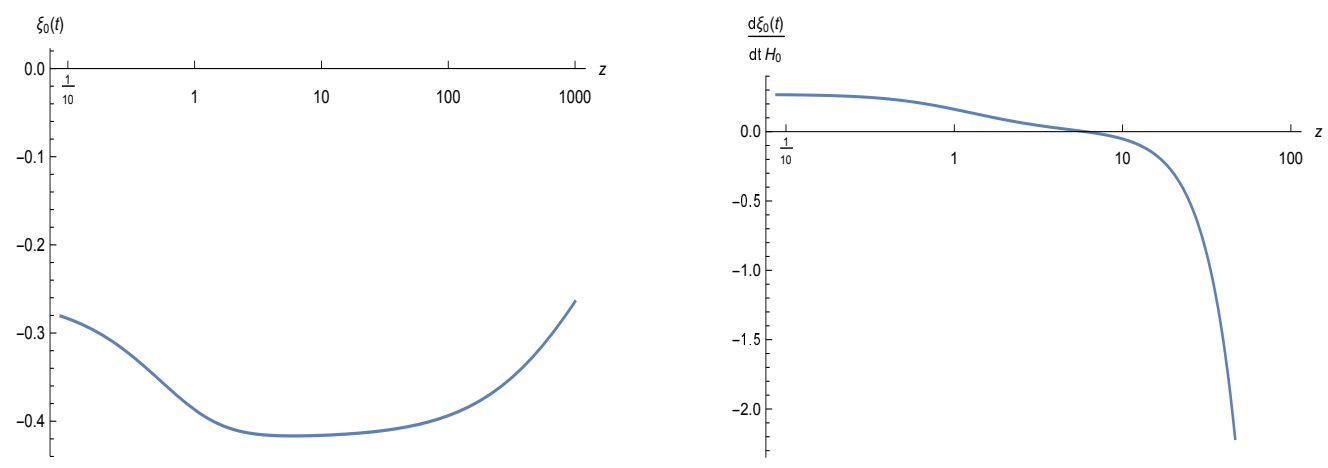

Figure 3: These graphs show $\xi_{0}(t)$ (left) and $\dot{\xi}_{0}(t) / H_{0}$ (right) as functions of the redshift $z$. Although $\xi_{0}(t)$ is negative definite, the sign of $\dot{\xi}_{0}(t) / H_{0}$ is positive for $z_{*}<z \lesssim 6$ and negative for $6 \lesssim z<\infty$. 

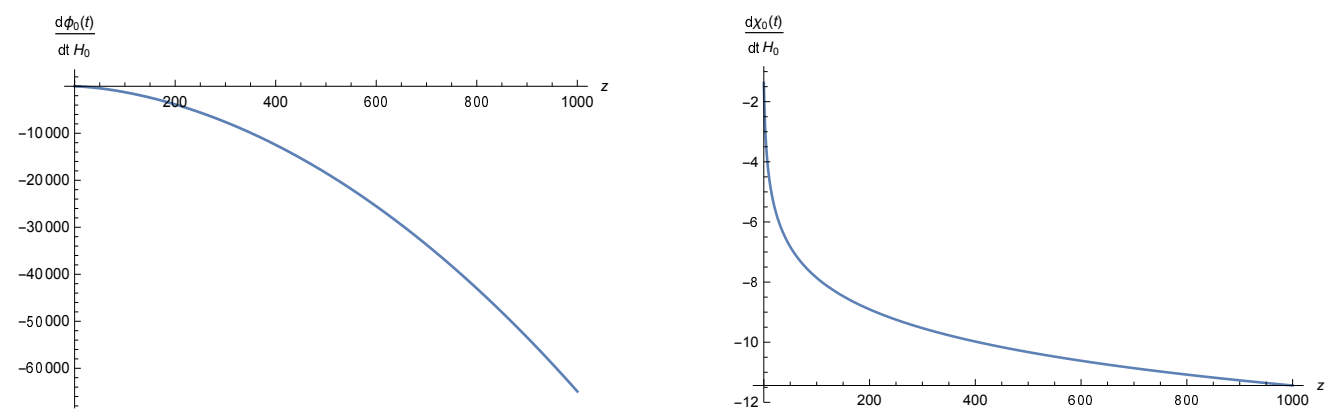

Figure 4: These graphs show $\dot{\phi}_{0}(t) / H_{0}$ (left) and $\dot{\chi}_{0}(t) / H_{0}$ (right) as functions of the redshift $z$. The function $\phi_{0}$ vanishes at $z=z_{*}$; it is negative and monotonically decreasing for $z_{*}<z<1000$. The function $\dot{\chi}_{0}$ is positive and monotonically decreasing for $z_{*} \geq z \geq 1000$.

\section{$3 \quad$ Linearized Scalar Perturbations}

The purpose of this section is to give the equations for linearized, scalar perturbations about the cosmological background of the previous section. We begin by reviewing the well known geometrical relations, then move to giving the equations for the auxiliary scalars and for the geometry. Finally, the various relations are specialized to the sub-horizon regime in which the comoving wavelength is much smaller than the Hubble length. To simplify the many tedious manipulations we work in units for which $c=1$.

\subsection{Perturbed Geometry}

In Newtonian gauge the geometry of linearized scalar perturbations is,

$$
g_{\mu \nu}(t, \vec{x}) d x^{\mu} d x^{\nu}=-[1+2 \widetilde{\Psi}(t, \vec{x})] d t^{2}+a^{2}(t)[1+2 \widetilde{\Phi}(t, \vec{x})] d \vec{x} \cdot d \vec{x} .
$$

Of course the perturbation fields can be decomposed into spatial plane waves,

$$
\widetilde{\Psi}(t, \vec{x}) \equiv \int \frac{d^{3} k}{(2 \pi)^{3}} e^{i \vec{k} \cdot \vec{x}} \Psi(t, \vec{k}) \quad, \quad \widetilde{\Phi}(t, \vec{x}) \equiv \int \frac{d^{3} k}{(2 \pi)^{3}} e^{i \vec{k} \cdot \vec{x}} \Phi(t, \vec{k}) .
$$

Because we are only interested in the linearized evolution equations each plane wave mode can be considered separately, and we will only report results

for these Fourier components. For example, $\delta \Gamma^{\rho}{ }_{\mu \nu}(t, \vec{k})$ stands for the spatial 
Fourier transform of the perturbed affine connection whose position-space result is,

$$
\delta \widetilde{\Gamma}_{\mu \nu}^{\rho}(t, \vec{x}) \equiv \int \frac{d^{3} k}{(2 \pi)^{3}} e^{i \vec{k} \cdot \vec{x}} \delta \Gamma_{\mu \nu}^{\rho}(t, \vec{k}) .
$$

To economize on space we will typically suppress the arguments $t$ and $\vec{k}$.

The $3+1$ decomposition of the perturbed affine connection is,

$$
\begin{array}{r}
\delta \Gamma_{00}^{0}=\dot{\Psi} \quad, \quad \delta \Gamma_{0 i}^{0}=i k_{i} \Psi \quad, \quad \delta \Gamma_{i j}^{0}=a^{2} \delta_{i j}[2 H(\Phi-\Psi)+\dot{\Phi}], \\
\delta \Gamma_{00}^{i}=\frac{i k_{i}}{a^{2}} \Psi \quad, \quad \delta \Gamma_{0 j}^{i}=\delta_{i j} \dot{\Phi} \quad, \quad \delta \Gamma_{j k}^{i}=i\left[\delta_{i j} k_{k}+\delta_{i k} k_{j}-\delta_{j k} k_{i}\right] \Phi .
\end{array}
$$

The corresponding $3+1$ decomposition of the perturbed Ricci tensor is,

$$
\begin{aligned}
\delta R_{00} & =-6 H \dot{\Phi}+3 H \dot{\Psi}-3 \ddot{\Phi}-\frac{k^{2}}{a^{2}} \Psi \\
\delta R_{0 i} & =2 i k_{i}[H \Psi-\dot{\Phi}] \\
\delta R_{i j} & =a^{2} \delta_{i j}\left[2\left(\dot{H}+3 H^{2}\right)(\Phi-\Psi)+6 H \dot{\Phi}-H \dot{\Psi}+\ddot{\Phi}+\frac{k^{2}}{a^{2}} \Phi\right]+k_{i} k_{j}(\Phi+\Psi) .
\end{aligned}
$$

The associated perturbed Ricci scalar is,

$$
\delta R=-12\left(\dot{H}+2 H^{2}\right) \Psi+24 H \dot{\Phi}-6 H \dot{\Psi}+6 \ddot{\Phi}+\frac{k^{2}}{a^{2}}(4 \Phi+2 \Psi) .
$$

And $3+1$ decomposing the perturbed Einstein tensor gives,

$$
\begin{aligned}
\delta G_{00}= & 6 H \dot{\Phi}+2 \frac{k^{2}}{a^{2}} \Phi, \\
\delta G_{0 i}= & 2 i k_{i}[H \Psi-\dot{\Phi}], \\
\delta G_{i j}= & a^{2} \delta_{i j}\left[-2\left(2 \dot{H}+3 H^{2}\right)(\Phi-\Psi)\right. \\
& \left.\quad-6 H \dot{\Phi}+2 H \dot{\Psi}-2 \ddot{\Phi}-\frac{k^{2}}{a^{2}}(\Phi+\Psi)\right]+k_{i} k_{j}(\Phi+\Psi) .
\end{aligned}
$$

We must also consider covariant derivatives of scalars, vectors and tensors whose background plus perturbed forms are,

$$
\begin{aligned}
S(t, \vec{x}) & =\bar{S}(t)+\delta S(t) e^{i \vec{k} \cdot \vec{x}}, \\
V^{\mu}(t, \vec{x}) & =\bar{V}(t) \delta^{\mu}{ }_{0}+\delta V^{\mu}(t) e^{i \vec{k} \cdot \vec{x}}, \\
\tau_{\mu \nu}(t, \vec{x}) & =\bar{\tau}(t) \delta^{0}{ }_{\mu} \delta^{0}{ }_{\nu}+\delta \tau_{\mu \nu}(t) e^{i \vec{k} \cdot \vec{x}} .
\end{aligned}
$$


Of course the scalar results are simplest,

$$
\begin{aligned}
\delta\left(\partial_{0} S\right) & =\delta \dot{S} \quad, \quad \delta\left(\partial_{i} S\right)=i k_{i} \delta S, \\
\delta\left(D_{0} D_{0} S\right) & =-\dot{\Psi} \dot{\bar{S}}+\delta \ddot{S} \\
\delta\left(D_{0} D_{i} S\right) & =i k_{i}\left[-\Psi \dot{\bar{S}}+\left(\partial_{t}-H\right) \delta S\right]=\delta\left(D_{i} D_{0} S\right) \\
\delta\left(D_{i} D_{j} S\right) & =-a^{2} \delta_{i j}[2 H(\Phi-\Psi)+\dot{\Phi}] \dot{S}-a^{2} \delta_{i j} H \delta \dot{S}-k_{i} k_{j} \delta S .
\end{aligned}
$$

Combining relations (61), (63) and background results implies,

$$
\delta(\square S)=2 \Psi[\ddot{\bar{S}}+3 H \dot{\bar{S}}]+[\dot{\Psi}-3 \dot{\Phi}] \dot{\bar{S}}-\left[\partial_{t}^{2}+3 H \partial_{t}+\frac{k^{2}}{a^{2}}\right] \delta S .
$$

Homogeneity and isotropy imply that the spatial part of vector perturbation takes the form,

$$
\delta V^{i} \equiv \frac{i k_{i}}{a^{2}} \delta V
$$

The various perturbed first covariant derivatives are,

$$
\begin{gathered}
\delta\left(D_{0} V^{0}\right)=\dot{\Psi} \bar{V}+\delta \dot{V}^{0}, \delta\left(D_{i} V^{0}\right)=i k_{i}\left[\Psi \bar{V}+\delta V^{0}+H \delta V\right] \\
\delta\left(D_{0} V^{j}\right)=\frac{i k_{j}}{a^{2}}\left[\Psi \bar{V}+\left(\partial_{t}-H\right) \delta V\right], \delta\left(D_{i} V^{j}\right)=\delta_{i j}\left[\dot{\Phi} \bar{V}+H \delta V^{0}\right]-\frac{k_{i} k_{j}}{a^{2}} \delta V
\end{gathered}
$$

Combining results gives the perturbed divergence,

$$
\delta\left(D_{\mu} V^{\mu}\right)=\bar{V}(3 \dot{\Phi}+\dot{\Psi})+\left(\partial_{t}+3 H\right) \delta V^{0}-\frac{k^{2}}{a^{2}} \delta V .
$$

It turns out that we do not require the perturbed second covariant derivatives.

Perturbed tensor covariant derivatives can become very complicated so it is best to restrict both the form of the perturbation and the derivatives we need. The point is to perturb the second derivative terms on the 3rd line of equation (5), so the tensor is $\tau_{\mu \nu}=\xi u_{\mu} u_{\nu}$. Because the spatial components $u_{i}$ are already first order, we can specialize to a perturbation of the form,

$$
\delta \tau_{00} \quad, \quad \delta \tau_{0 i}=\delta \tau_{i 0}=i k_{i} \delta \tau_{0} \quad, \quad \delta \tau_{i j}=0
$$

And the perturbed derivative we require is,

$$
\mathcal{T}_{\mu \nu} \equiv \delta\left(-\square \tau_{\mu \nu}-g_{\mu \nu} D_{\alpha} D_{\beta} \tau^{\alpha \beta}+2 D_{\alpha} D_{(\mu} \tau_{\nu)}{ }^{\alpha}\right)
$$


Homogeneity and isotropy restrict the form of $\mathcal{T}_{\mu \nu}$,

$$
\mathcal{T}_{00} \quad, \quad \mathcal{T}_{0 i}=\mathcal{T}_{i 0}=i k_{i} \mathcal{T}_{0} \quad, \quad \mathcal{T}_{i j}=a^{2} \delta_{i j} \mathcal{T}-k_{i} k_{j} \Delta \mathcal{T}
$$

In Appendix A we derive the following results for the four components of relation (71),

$$
\begin{gathered}
\mathcal{T}_{00}=3\left(\partial_{t}+6 H\right)(\bar{\tau} \dot{\Phi})-3 H \bar{\tau} \dot{\Psi}-6\left(\partial_{t}+3 H\right)(H \bar{\tau} \Psi)-\frac{k^{2}}{a^{2}} \bar{\tau} \Psi \\
+3\left(\partial_{t}+3 H\right)\left(H \delta \tau_{00}\right)+\frac{k^{2}}{a^{2}}\left[\delta \tau_{00}+4 H \delta \tau_{0}\right], \\
\mathcal{T}_{0}=2 \bar{\tau}\left(\partial_{t}+H\right) \Psi+3 \dot{\bar{\tau}} \Psi-\left(\partial_{t}+H\right) \delta \tau_{00}+2\left(\dot{H}+3 H^{2}\right) \delta \tau_{0} \\
\mathcal{T}=-[\bar{\tau} \ddot{\Phi}+4 \dot{\bar{\tau}} \dot{\Phi}+6 H \bar{\tau} \dot{\Phi}]+[2 \bar{\tau} \ddot{\Psi}+5 \dot{\bar{\tau}} \dot{\Psi}+9 H \bar{\tau} \dot{\Psi}]+\frac{k^{2}}{a^{2}} \bar{\tau} \Psi \\
-2(\Phi-2 \Psi)\left(\partial_{t}+3 H\right)\left(\partial_{t}+H\right) \bar{\tau}-\left(\partial_{t}+3 H\right)\left(\partial_{t}+H\right) \delta \tau_{00}-\frac{2 k^{2}}{a^{2}} \partial_{t} \delta \tau_{0} \\
\Delta \mathcal{T}=-2\left(\partial_{t}+H\right) \delta \tau_{0} .
\end{gathered}
$$

\subsection{Perturbed Auxiliary Scalar Equations}

Let us first note from equation (2) that the components of the perturbed 4-velocity are,

$$
\delta u^{0}=-\Psi \quad, \quad \delta u^{i}=\frac{-i k_{i} \delta \chi}{a^{2} \dot{\chi}_{0}} .
$$

Now apply relations (50) and (64) to (3) to infer the equation for $\delta \phi$,

$$
\left[\partial_{t}^{2}+3 H \partial_{t}+\frac{k^{2}}{a^{2}}\right] \delta \phi=\left[6 \partial_{t}+12 H-3 \dot{\phi}_{0}\right] \dot{\Phi}+\left[-6 H \partial_{t}+\dot{\phi}_{0} \partial_{t}+\frac{2 k^{2}}{a^{2}}\right] \Psi .
$$

Note that this gives us the perturbation in $Z$,

$$
\delta Z=2 Z_{0}\left[\frac{\delta \dot{\phi}}{\dot{\phi}_{0}}-\Psi\right] .
$$

The equation for $\delta \chi$ requires only relations (64) and (3),

$$
\left[\partial_{t}^{2}+3 H \partial_{t}+\frac{k^{2}}{a^{2}}\right] \delta \chi=-3 \dot{\chi}_{0} \dot{\Phi}+\left[\dot{\chi}_{0} \partial_{t}+2\right] \Psi
$$


Using relations (64) and (68) in (44) gives the equation for $\delta \xi$,

$$
\left[\partial_{t}^{2}+3 H \partial_{t}+\frac{k^{2}}{a^{2}}\right] \delta \xi=2\left(\partial_{t}+3 H\right)\left[f_{y}^{\prime}\left(Z_{0}\right) \delta \dot{\phi}+f_{y}^{\prime \prime}\left(Z_{0}\right) \dot{\phi}_{0} \delta Z\right]+\frac{2 k^{2}}{a^{2}} f_{y}^{\prime}\left(Z_{0}\right) \delta \phi .
$$

And our equation for $\delta \psi$ comes from using relations (51), (64) and (68) in (4),

$$
\left[\partial_{t}^{2}+3 H \partial_{t}+\frac{k^{2}}{a^{2}}\right] \delta \psi=\frac{k^{2}}{a^{2}} \frac{8 \xi_{0}}{\dot{\chi}_{0}}\left[\frac{\dot{H} \delta \chi}{\dot{\chi}_{0}}+H \Psi-\dot{\Phi}\right] .
$$

\subsection{Perturbed Gravitational Field Equations}

We employ the following notation to express the modified Einstein equation,

$$
\mathcal{E}_{\mu \nu} \equiv G_{\mu \nu}+\mathcal{G}_{\mu \nu}=8 \pi G T_{\mu \nu}
$$

Here $G_{\mu \nu}$ the usual Einstein tensor, $\mathcal{G}_{\mu \nu}$ is the MOND correction to it given in equation (5) and $T_{\mu \nu}$ is the stress tensor without dark matter. Relations (28) and (29) give the nonzero components of (82) when the geometry is specialized to a cosmological background. We denote the first order perturbations of $\mathcal{E}_{\mu \nu}$ and $T_{\mu \nu}$ as,

$$
\begin{gathered}
\delta \mathcal{E}_{00}, \delta \mathcal{E}_{0 i}=\delta \mathcal{E}_{i 0}=i k_{i} \delta \mathcal{E}_{0} \quad, \quad \delta \mathcal{E}_{i j}=a^{2} \delta_{i j} \delta \mathcal{E}-k_{i} k_{j} \Delta \mathcal{E} \\
\delta T_{00}=\delta \rho \quad, \quad \delta T_{0 i}=\delta T_{i 0}=i k_{i} \Delta \rho \quad, \quad \delta T_{i j}=a^{2} \delta_{i j} \delta T .
\end{gathered}
$$

The various components of the perturbed stress tensor are related by the two conservation equations,

$$
\begin{aligned}
& 0=-\delta \dot{\rho}-3 H(\delta \rho+\delta T)+\frac{k^{2}}{a^{2}} \Delta \rho-3\left(\rho_{0}+p_{0}\right) \dot{\Phi}+2 \rho_{0} \dot{\Psi}+6 H p_{0}(\Phi-\Psi), \\
& 0=-\left(\partial_{t}+3 H\right) \Delta \rho+\delta T-2 p_{0} \Phi+\left(\rho_{0}+p_{0}\right) \Psi
\end{aligned}
$$

Similar relations hold for the components of $\delta \mathcal{E}_{\mu \nu}$. Because $\delta \mathcal{E}$ follows from conservation we will not report it.

Relations (71-75) give the perturbed second covariant derivatives of a tensor $\tau_{\mu \nu}=\xi u_{\mu} u_{\nu}$. From expression (76) we see that the components (69) of this tensor are,

$$
\bar{\tau}=\xi_{0} \quad, \quad \delta \tau_{00}=2 \Psi \xi_{0}+\delta \xi \quad, \quad \delta \tau_{0}=\frac{\xi_{0} \delta \chi}{\dot{\chi}_{0}}
$$


From the 00 component of (15), with relations (150), (154), (172) and (87) we infer the perturbed 00 equation,

$$
\begin{aligned}
{[6 H} & \left.+3 \dot{\xi}_{0}+12 H \xi_{0}\right] \dot{\Phi}+a_{0}^{2} f_{y}\left(Z_{0}\right) \Psi+a_{0}^{2}\left[\frac{f_{y}^{\prime}\left(Z_{0}\right)}{2}-Z_{0} f_{y}^{\prime \prime}\left(Z_{0}\right)\right] \delta Z+\frac{\dot{\xi}_{0} \delta \dot{\phi}-\dot{\phi}_{0} \delta \dot{\xi}}{2} \\
& +\left[3 H \partial_{t}+6 H^{2}\right] \delta \xi-\left[\dot{\chi}_{0} \partial_{t}-1\right] \frac{\delta \psi}{2}+\frac{k^{2}}{a^{2}}\left[2 \Phi+\delta \xi+\frac{4 H \xi_{0} \delta \chi}{\dot{\chi}_{0}}\right]=8 \pi G \delta \rho .(88)
\end{aligned}
$$

The perturbed $0 i$ components follow from using (151), (55), (173) and (87) in expression (5),

$$
\left(2 H+4 H \xi_{0}+\dot{\xi}_{0}\right) \Psi-\left(2+4 \xi_{0}\right) \dot{\Phi}-\left(\partial_{t}+H+\frac{1}{2} \dot{\phi}_{0}\right) \delta \xi-\frac{1}{2} \dot{\chi}_{0} \delta \psi=8 \pi G \Delta \rho .
$$

Finally, expressions (56) and (75) give us the gravitational slip equation,

$$
\Phi+\Psi+2\left(\partial_{t}+H\right)\left[\frac{\xi_{0} \delta \chi}{\dot{\chi}_{0}}\right]=0 .
$$

\subsection{The Sub-Horizon Regime}

The perturbed equations of the previous two sub-sections simplify dramatically in the sub-horizon regime of $k \gg H a$. In this regime time derivatives of the fields are also negligible so the various auxiliary scalar equations (77), (79), (80) and (81) can be used to express the scalar perturbations in terms of the two gravitational potentials,

$$
k \gg H a \Longrightarrow \delta \phi=2 \Psi, \delta \chi=0, \delta \xi=4 f_{y}^{\prime}\left(Z_{0}\right) \Psi, \delta \psi=\frac{8 \xi_{0}}{\dot{\chi}_{0}}[H \Psi-\dot{\Phi}]
$$

Making the same approximations in the 00 component (88) of the perturbed gravitational field equations implies,

$$
k \gg H a \quad \Longrightarrow \quad \frac{k^{2}}{a^{2}}\left[2 \Phi+4 f_{y}^{\prime}\left(Z_{0}\right) \Psi\right]=8 \pi G \delta \rho
$$

The $0 i$ equation (89) reduces to,

$$
k \gg H a \quad \Longrightarrow \quad 2 H \Psi-2 \dot{\Phi}-4\left(\partial_{t}+H\right)\left[f_{y}^{\prime}\left(Z_{0}\right) \Psi\right]=8 \pi G \Delta \rho .
$$

And the gravitational slip equation (90) reduces to that of unmodified general relativity,

$$
k \gg H a \quad \Longrightarrow \quad \Phi+\Psi=0 \text {. }
$$


Relations (92) and (94) are fatal because the first term in the square brackets of (92) $-2 \Phi-$ is the contribution from unmodified general relativity, whereas the second term - $4 f_{y}^{\prime}\left(Z_{0}\right) \Psi=-4 f_{y}^{\prime}\left(Z_{0}\right) \Phi$ using (94) - is the MOND correction. The right hand side of (92) is the perturbed energy density without dark matter. For this model to be viable the MOND correction must largely cancel the factor of $2 \Phi$ from general relativity, so that one gets the same gravitational response from the much smaller source. However, a glance at Fig. 2] reveals that the function $f_{y}^{\prime}\left(Z_{0}\right) \lesssim 0.015$ is much too small, and actually strengthens the general relativistic result for $z \lesssim 6$. Hence the gravitational response to matter perturbations is much too weak.

\section{Discussion}

In this paper we have considered structure formation in a nonlocal, metricbased realization of MOND [30, 31]. This model involves augmenting the gravitational action by an algebraic function $f_{y}(Z)$ of a nonlocal invariant. The function $f_{y}(Z)$ is constrained for positive $Z$ by the Tully-Fisher relation (for $0<Z \ll 1$ ) and by the need to leave solar system results undisturbed (for $1 \lesssim Z$ ). Cosmology corresponds to $Z<0$ and the function was chosen to reproduce the $\Lambda \mathrm{CDM}$ expansion history (until very late times) without dark matter [32].

Section 2 was devoted to describing the model in general, and as specialized to static, spherically symmetric geometries and to homogeneous and isotropic geometries. In section 3 we derived the equations - (77) auxiliary scalars and (88,90) for the gravitational potentials - governing first order scalar perturbations around the cosmological background. Specializing those equations to the sub-horizon regime leads to vastly simpler equations - (91) for the auxiliary scalars and (92 94) for the gravitational potentials. These simplified equations show that the MOND corrections cannot possibly make up for the absence of dark matter in structure formation. Hence this particular model is falsified.

It is interesting to contrast our negative result with what happens in a nonlocal cosmology model that was proposed explain cosmic acceleration without dark energy [39]. This model cannot reproduce MOND [40] but its free function $f(X)$ (of a different nonlocal invariant) can be adjusted to enforce the $\Lambda \mathrm{CDM}$ expansion history [41] without a cosmological constant. However, when linearized scalar perturbations are studied in nonlocal cos- 
mology [42, 43], the "extra" terms which are not present in general relativity turn out to be significant, and actually cause the model to agree better with data [4, 45. For both nonlocal cosmology and nonlocal MOND, the "extra" terms are proportional to the derivative of the free function - $f^{\prime}\left(X_{0}\right)$ for nonlocal cosmology and $f_{y}^{\prime}\left(Z_{0}\right)$ for nonlocal MOND. The key distinction between the two models is that $f^{\prime}\left(X_{0}\right)$ is significant for nonlocal cosmology, whereas $f_{y}^{\prime}\left(Z_{0}\right)$ is nearly zero for nonlocal MOND. That seems to be reason why the results are so very different.

That $f_{y}^{\prime}\left(Z_{0}\right)$ must be small for very large redshift follows from the asymptotic relation (42), which could be written,

$$
f_{y}^{\prime}\left(Z_{0}\right) \longrightarrow \frac{1}{44} \times \frac{\Omega_{c}}{\Omega_{m}} \times\left(\frac{1+z_{\mathrm{eq}}}{1+z}\right) \simeq 0.019 \times\left(\frac{3300}{1+z}\right) .
$$

However, this relation only pertains for $z>z_{e q}$, during the radiation dominated phase when matter is unimportant. There is no simple way to understand the crucial behavior of $f_{y}^{\prime}\left(Z_{0}\right)$ for smaller redshift, which is shown in Fig. 2. The fact that $f_{y}\left(Z_{0}\right)$ approaches the limiting form (95) from below, and actually goes to negative infinity at $z=z_{*}$, seems to follow from our decision to solve (35) with the initial condition $f\left(z_{*}\right)=0$. In retrospect, it might be more reasonable to impose the condition $f^{\prime}\left(z_{*}\right)=0$, which would keep $f_{y}^{\prime}\left(Z_{0}\right)$ finite at $z=z_{*}$, and might result in $f_{y}^{\prime}\left(Z_{0}\right)$ approaching the limiting form (95) from above. This would be the simplest fix because it would leave the equations for linearized perturbations unchanged when written in terms of the generic background quantities, changing only the numerical values of those background quantities.

Two more complicated fixes are also conceivable. The first would be to involve another invariant as suggested in the original proposal [30] for nonlocal MOND. This extra invariant is not required to reproduce either the Tully-Fisher relation, or (most of) the $\Lambda$ CDM expansion history, but perhaps it plays a crucial role in structure formation.

The second conceivable extension of the model would be to make $a_{0}$ dynamical. The numerical coincidence that $a_{0}$ is about $c H_{0} / 2 \pi$ has led many to suspect that $a_{0}$ is not actually a new constant but rather a functional of the geometry which is always close to $c$ times the Hubble parameter [46, 47]. The idea would be to keep cosmology in the deep MOND regime and NOT use the function $f_{y}(Z)$ to enforce the $\Lambda$ CDM expansion history. 


\section{Appendix: Perturbed Tensor Derivatives}

The purpose of this appendix is to derive relations (72) 75 . Our strategy is to construct the final answer in three steps:

1. Expand $\mathcal{T}_{\mu \nu}$ in terms of $\delta g_{\mu \nu}$ and $\delta\left(D_{\rho} D_{\sigma} \tau_{\mu \nu}\right)$;

2. Expand $\delta\left(D_{\rho} D_{\sigma} \tau_{\mu \nu}\right)$ in terms of $\delta \Gamma^{\rho}{ }_{\mu \nu}$ and $\delta\left(D_{\rho} \tau_{\mu \nu}\right)$; and

3. Expand $\delta\left(D_{\rho} \tau_{\mu \nu}\right)$ in terms of $\delta \Gamma_{\mu \nu}^{\rho}$ and $\delta \tau_{\mu \nu}$.

Because many cancellations occur at each level we combine terms in $\mathcal{T}_{00}$, $\mathcal{T}_{0 i}$ and $\mathcal{T}_{i j}$ after each expansion, before proceeding to the next step. The nonzero components of the background quantities we require are,

$$
\begin{aligned}
& \bar{\Gamma}_{i j}^{0}=H a^{2} \delta_{i j}, \quad \bar{\Gamma}_{0 j}^{i}=H \delta_{i j}, \\
& \overline{D_{0} \tau_{00}}=\dot{\bar{\tau}}, \overline{D_{i} \tau_{0 j}}=-H \bar{\tau} a^{2} \delta_{i j}, \\
& \overline{D_{0} D_{0} \tau_{00}}=\ddot{\bar{\tau}} \quad, \quad \overline{D_{i} D_{j} \tau_{00}}=-H\left(\partial_{t}-2 H\right) \bar{\tau} a^{2} \delta_{i j}, \\
& \overline{D_{0} D_{i} \tau_{0 j}}=-\partial_{t}(H \bar{\tau}) a^{2} \delta_{i j} \quad, \quad \overline{D_{i} D_{0} \tau_{0 j}}=-H\left(\partial_{t}-H\right) \bar{\tau} a^{2} \delta_{i j},
\end{aligned}
$$

and

$$
\overline{D_{i} D_{j} \tau_{k \ell}}=H^{2} \bar{\tau} a^{4}\left(\delta_{i k} \delta_{j \ell}+\delta_{i \ell} \delta_{j k}\right) .
$$

The first step expansions are:

$$
\begin{aligned}
& -\delta\left(\square \tau_{\mu \nu}\right)=-2 \Psi \overline{D_{0} D_{0} \tau_{\mu \nu}}+\frac{2 \Phi}{a^{2}} \overline{D_{k} D_{k} \tau_{\mu \nu}} \\
& +\delta\left(D_{0} D_{0} \tau_{\mu \nu}\right)-\frac{1}{a^{2}} \delta\left(D_{k} D_{k} \tau_{\mu \nu}\right), \\
& -\delta\left(g_{\mu \nu} D_{\alpha} D_{\beta} \tau^{\alpha \beta}\right)=\delta g_{\mu \nu}\left[-\overline{D_{0} D_{0} \tau_{00}}+\frac{\left(\overline{D_{0} D_{k} \tau_{0 k}}+\overline{D_{k} D_{0} \tau_{k 0}}\right)}{a^{2}}-\frac{\overline{D_{k} D_{\ell} \tau_{k \ell}}}{a^{4}}\right] \\
& +\overline{g_{\mu \nu}}\left[4 \Psi \overline{D_{0} D_{0} \tau_{00}}-\frac{2(\Psi+\Phi)}{a^{2}}\left(\overline{D_{0} D_{k} \tau_{0 k}}+\overline{D_{k} D_{0} \tau_{k 0}}\right)+\frac{4 \Phi}{a^{4}} \overline{D_{k} D_{\ell} \tau_{k \ell}}\right. \\
& \left.-\delta\left(D_{0} D_{0} \tau_{00}\right)+\frac{1}{a^{2}} \delta\left(D_{0} D_{k} \tau_{0 k}+D_{k} D_{0} \tau_{k 0}\right)-\frac{1}{a^{4}} \delta\left(D_{k} D_{\ell} \tau_{k \ell}\right)\right], \\
& \delta\left(2 D_{\alpha} D_{(\mu} \tau_{\nu)}{ }^{\alpha}\right)=4 \Psi \overline{D_{0} D_{(\mu} \tau_{\nu) 0}}-\frac{4 \Phi}{a^{2}} \overline{D_{k} D_{(\mu} \tau_{\nu) k}} \\
& -2 \delta\left(D_{0} D_{(\mu} \tau_{\nu) 0}\right)+\frac{2}{a^{2}} \delta\left(D_{k} D_{(\mu} \tau_{\nu) k}\right) .
\end{aligned}
$$


The $3+1$ totals after the 1 st step are,

$$
\begin{aligned}
\mathcal{T}_{00}= & -6 H\left(\partial_{t}+7 H\right)(H \bar{\tau}) \Phi+24 H^{2} \bar{\tau} \Psi \\
& +\frac{1}{a^{2}} \delta\left(-D_{k} D_{k} \tau_{00}-D_{0} D_{k} \tau_{0 k}+D_{k} D_{0} \tau_{0 k}\right)+\frac{\delta\left(D_{k} D_{\ell} \tau_{k \ell}\right)}{a^{4}}, \\
\mathcal{T}_{0 i}= & -\delta\left(D_{0} D_{i} \tau_{00}\right)+\frac{1}{a^{2}} \delta\left(-D_{k} D_{k} \tau_{0 i}+D_{k} D_{0} \tau_{i k}+D_{k} D_{i} \tau_{0 k}\right) \\
\mathcal{T}_{i j}= & a^{2} \delta_{i j}\left[\left(-2 \ddot{\bar{\tau}}+12 H^{2} \bar{\tau}\right) \Phi+\left(4 \ddot{\bar{\tau}}+8 H \dot{\bar{\tau}}+\left(2 \dot{H}-6 H^{2}\right) \bar{\tau}\right) \Psi\right. \\
& \left.-\delta\left(D_{0} D_{0} \tau_{00}\right)+\frac{1}{a^{2}} \delta\left(D_{0} D_{k} \tau_{0 k}+D_{k} D_{0} \tau_{0 k}\right)-\frac{1}{a^{4}} \delta\left(D_{k} D_{\ell} \tau_{k \ell}\right)\right] \\
& +\delta\left(D_{0} D_{0} \tau_{i j}-2 D_{0} D_{(i} \tau_{j) 0}\right)+\frac{1}{a^{2}} \delta\left(-D_{k} D_{k} \tau_{i j}+2 D_{k} D_{(i} \tau_{j) k}\right) .
\end{aligned}
$$

Note that only the terms on the last line of (106) can possibly contribute to the $k_{i} k_{j}$ part of $\mathcal{T}_{i j}$.

Of course it is only necessary to implement the second step for the $\delta\left(D_{\rho} D_{\sigma} \tau_{\mu \nu}\right)$ parts of expressions (104-106). The key expansion is,

$$
\begin{aligned}
& \delta\left(D_{\rho} D_{\sigma} \tau_{\mu \nu}\right)=-\delta \Gamma_{\rho \sigma}^{\alpha} \overline{D_{\alpha} \tau_{\mu \nu}}-\delta \Gamma_{\rho \mu}^{\alpha} \overline{D_{\sigma} \tau_{\alpha \nu}}-\delta \Gamma_{\rho \nu}^{\alpha} \overline{D_{\sigma} \tau_{\mu \alpha}} \\
& \quad+\partial_{\rho} \delta\left(D_{\sigma} \tau_{\mu \nu}\right)-\bar{\Gamma}_{\rho \sigma}^{\alpha} \delta\left(D_{\alpha} \tau_{\mu \nu}\right)-\bar{\Gamma}_{\rho \mu}^{\alpha} \delta\left(D_{\sigma} \tau_{\alpha \nu}\right)-\bar{\Gamma}_{\rho \nu}^{\alpha} \delta\left(D_{\sigma} \tau_{\mu \alpha}\right) .
\end{aligned}
$$

The $\delta\left(D_{\rho} D_{\sigma} \tau_{\mu \nu}\right)$ part of $\mathcal{T}_{00}$ from expression (104) gives,

$$
\begin{aligned}
& \frac{1}{a^{2}} \delta\left(-D_{k} D_{k} \tau_{00}\right.\left.-D_{0} D_{k} \tau_{0 k}+D_{k} D_{0} \tau_{0 k}\right)+\frac{\delta\left(D_{k} D_{\ell} \tau_{k \ell}\right)}{a^{4}} \\
&=3 H \bar{\tau}[(\dot{\Phi}-\dot{\Psi})+8 H(\Phi-\Psi)]-\frac{1}{a^{2}}\left(\partial_{0}+H\right) \delta\left(D_{k} \tau_{0 k}\right) \\
& \quad-\frac{2 H}{a^{2}} \delta\left(D_{0} \tau_{k k}\right)+\frac{i k_{k}}{a^{2}} \delta\left(D_{0} \tau_{0 k}-D_{k} \tau_{00}\right)+\frac{i k_{k}}{a^{4}} \delta\left(D_{\ell} \tau_{k \ell}\right) .
\end{aligned}
$$

The step 1 reduction of $\mathcal{T}_{0 i}$ in expression (105) contains only $\delta\left(D_{\rho} D_{\sigma} \tau_{\mu \nu}\right)$ terms,

$$
\begin{aligned}
\mathcal{T}_{0 i}= & i k_{i}(\dot{\bar{\tau}}-2 H \bar{\tau}) \Psi-\left(\partial_{0}+H\right) \delta\left(D_{i} \tau_{00}\right) \\
& +\frac{i k_{k}}{a^{2}} \delta\left(-D_{k} \tau_{0 i}+D_{0} \tau_{i k}+D_{i} \tau_{0 k}\right)-2 H \delta\left(D_{0} \tau_{0 i}\right)-\frac{H}{a^{2}} \delta\left(D_{i} \tau_{k k}\right) .
\end{aligned}
$$

The step 1 reduction of $\mathcal{T}_{i j}$ in expression (106) contained some terms which are already proportional to $a^{2} \delta_{i j}$,

$$
-\delta\left(D_{0} D_{0} \tau_{00}\right)+\frac{1}{a^{2}} \delta\left(D_{0} D_{k} \tau_{0 k}+D_{k} D_{0} \tau_{0 k}\right)-\frac{1}{a^{4}} \delta\left(D_{k} D_{\ell} \tau_{k \ell}\right)
$$




$$
\begin{array}{r}
=-3(\dot{\bar{\tau}}+H \bar{\tau})(\dot{\Phi}-\dot{\Psi})-6 H(\dot{\bar{\tau}}+4 H \bar{\tau})(\Phi-\Psi)-\left(\partial_{0}+3 H\right) \delta\left(D_{0} \tau_{00}\right) \\
+\frac{1}{a^{2}}\left(\partial_{0}+H\right) \delta\left(D_{k} \tau_{0 k}\right)+\frac{i k_{k}}{a^{2}} \delta\left(D_{0} \tau_{0 k}\right)-\frac{i k_{k}}{a^{4}} \delta\left(D_{\ell} \tau_{k \ell}\right) .(110
\end{array}
$$

The other $\delta\left(D_{\rho} D_{\sigma} \tau_{\mu \nu}\right)$ terms in expression (106) are,

$$
\begin{aligned}
& \delta\left(D_{0} D_{0} \tau_{i j}-2 D_{0} D_{(i} \tau_{j) 0}\right)+\frac{1}{a^{2}} \delta\left(-D_{k} D_{k} \tau_{i j}+2 D_{k} D_{(i} \tau_{j) k}\right) \\
&=2 H \bar{\tau} a^{2} \delta_{i j}[(\dot{\Phi}-\dot{\Phi})+6 H(\Phi-\Psi)]+\left(\partial_{0}-H\right) \delta\left(D_{0} \tau_{i j}\right) \\
&-2\left(\partial_{0}+H\right) \delta\left(D_{i} \tau_{0 j}\right)+\frac{i k_{k}}{a^{2}} \delta\left[-D_{k} \tau_{i j}+2 D_{i} \tau_{j k}\right]
\end{aligned}
$$

As before, it is only necessary to implement the 3rd step reduction for those parts of expressions (108-111) which contain $\delta\left(D_{\rho} \tau_{\mu \nu}\right)$. The key reduction of step 3 is,

$$
\delta\left(D_{\rho} \tau_{\mu \nu}\right)=-\delta \Gamma_{\rho \mu}^{\sigma} \bar{\tau}_{\sigma \nu}-\delta \Gamma_{\rho \nu}^{\sigma} \bar{\tau}_{\mu \sigma}+\partial_{\rho} \delta \tau_{\mu \nu}-\bar{\Gamma}_{\rho \mu}^{\sigma} \delta \tau_{\sigma \nu}-\bar{\Gamma}_{\rho \nu}^{\sigma} \delta \tau_{\mu \sigma} .
$$

The step 3 reduction of the $\delta\left(D_{\rho} \tau_{\mu \nu}\right)$ terms in (108) is,

$$
\begin{gathered}
-\frac{1}{a^{2}}\left(\partial_{0}+H\right) \delta\left(D_{k} \tau_{0 k}\right)-\frac{2 H}{a^{2}} \delta\left(D_{0} \tau_{k k}\right)+\frac{i k_{k}}{a^{2}} \delta\left(D_{0} \tau_{0 k}-D_{k} \tau_{00}\right)+\frac{i k_{k}}{a^{4}} \delta\left(D_{\ell} \tau_{k \ell}\right) \\
=\left(\partial_{t}+3 H\right)[3 \bar{\tau} \dot{\Phi}+6 H \bar{\tau}(\Phi-\Psi)]-\frac{k^{2}}{a^{2}} \bar{\tau} \Psi \\
+3\left(\partial_{t}+3 H\right)\left(H \delta \tau_{00}\right)+\frac{k^{2}}{a^{2}}\left[\delta \tau_{00}+4 H \delta \tau_{0}\right] .(113)
\end{gathered}
$$

The step 3 reduction of the $\delta\left(D_{\rho} \tau_{\mu \nu}\right)$ terms in (109) is,

$$
\begin{array}{r}
\left(\partial_{0}+H\right) \delta\left(D_{i} \tau_{00}\right)-\frac{i k_{k}}{a^{2}} \delta\left(D_{k} \tau_{0 i}-D_{0} \tau_{i k}-D_{i} \tau_{0 k}\right)-2 H \delta\left(D_{0} \tau_{0 i}\right)-\frac{H}{a^{2}} \delta\left(D_{i} \tau_{k k}\right) \\
=i k_{i}\left[2\left(\partial_{t}+2 H\right)(\bar{\tau} \Psi)-\left(\partial_{t}+H\right) \delta \tau_{00}+\left(2 \dot{H}+6 H^{2}\right) \delta \tau_{0}\right] .(11)
\end{array}
$$

The step 3 reduction of the $\delta\left(D_{\rho} \tau_{\mu \nu}\right)$ terms in (110) is,

$$
\begin{array}{r}
-\left(\partial_{0}+3 H\right) \delta\left(D_{0} \tau_{00}\right)+ \\
=\left(\partial_{t}+3 H\right)[-3 \bar{\tau} \dot{\Phi}+2 \bar{\tau} \dot{\Psi}-6 H \bar{\tau}(\Phi-\Psi)]+\frac{k^{2}}{a^{2}} \bar{\tau} \Psi \\
-\left(\partial_{t}+3 H\right)^{2} \delta \tau_{00}-\frac{2 k^{2}}{a^{2}}\left(\partial_{t}+H\right) \delta \tau_{0} \cdot
\end{array}
$$


And the step 3 reduction of the $\delta\left(D_{\rho} \tau_{\mu \nu}\right)$ terms in (111) is,

$$
\begin{aligned}
& \left(\partial_{0}-H\right) \delta\left(D_{0} \tau_{i j}\right)-2\left(\partial_{0}+H\right) \delta\left(D_{i} \tau_{0 j}\right)+\frac{i k_{k}}{a^{2}} \delta\left[-D_{k} \tau_{i j}+2 D_{i} \tau_{j k}\right]=2 a^{2} \delta_{i j} \\
& \times\left[\left(\partial_{t}+3 H\right)\left[\bar{\tau} \dot{\Phi}+2 H \bar{\tau}(\Phi-\Psi)+H \delta \tau_{00}\right]+\frac{k^{2} H \delta \tau_{0}}{a^{2}}\right]+k_{i} k_{j} 2\left(\partial_{t}+H\right) \delta \tau_{0} .
\end{aligned}
$$

Combining expressions (104), (108) and (113) gives our final result for $\mathcal{T}_{00}$

$$
\begin{aligned}
\mathcal{T}_{00}=3\left(\partial_{t}+6 H\right)(\bar{\tau} \dot{\Phi})-3 H \bar{\tau} \dot{\Psi}-6\left(\partial_{t}+3 H\right)(H \bar{\tau} \Psi)-\frac{k^{2}}{a^{2}} \bar{\tau} \Psi \\
+3\left(\partial_{t}+3 H\right)\left(H \delta \tau_{00}\right)+\frac{k^{2}}{a^{2}}\left[\delta \tau_{00}+4 H \delta \tau_{0}\right]
\end{aligned}
$$

Our final result for $\mathcal{T}_{0}$ comes from expressions (109) and (114),

$$
\mathcal{T}_{0}=2 \bar{\tau}\left(\partial_{t}+H\right) \Psi+3 \dot{\bar{\tau}} \Psi-\left(\partial_{t}+H\right) \delta \tau_{00}+2\left(\dot{H}+3 H^{2}\right) \delta \tau_{0} .
$$

Combining expressions (106), (110), (111), (115) and (116) gives our result for $\mathcal{T}$

$$
\begin{aligned}
\mathcal{T}= & -[\bar{\tau} \ddot{\Phi}+4 \dot{\bar{\tau}} \dot{\Phi}+6 H \bar{\tau} \dot{\Phi}]+[2 \bar{\tau} \ddot{\Psi}+5 \dot{\bar{\tau}} \dot{\Psi}+9 H \bar{\tau} \dot{\Psi}]+\frac{k^{2}}{a^{2}} \bar{\tau} \Psi \\
& -2(\Phi-2 \Psi)\left(\partial_{t}+3 H\right)\left(\partial_{t}+H\right) \bar{\tau}-\left(\partial_{t}+3 H\right)\left(\partial_{t}+H\right) \delta \tau_{00}-\frac{2 k^{2}}{a^{2}} \partial_{t} \delta \tau_{0} \cdot(11
\end{aligned}
$$

And our result for $\Delta \mathcal{T}$ comes entirely from (116),

$$
\Delta \mathcal{T}=-2\left(\partial_{t}+H\right) \delta \tau_{0}
$$

The principal complication in expressions (117) and (119) is their dependence upon the potentials $\Phi$ and $\Psi$, and the perturbation $\delta \tau_{00}$, and their respective time derivatives. This can actually be predicted by transforming the background expressions,

$$
\overline{\mathcal{T}}_{00}=3\left(\partial_{t}+3 H\right)(H \bar{\tau}) \quad, \quad \overline{\mathcal{T}}_{i j}=-a^{2} \delta_{i j}\left(\partial_{t}+3 H\right)\left(\partial_{t}+H\right) \bar{\tau},
$$

by the same transformation which would carry the background geometry into the perturbed one assuming the potentials depend only on time,

$$
-d t^{2}+a^{2} d \vec{x} \cdot d \vec{x} \longrightarrow-[1+2 \Psi] d t^{2}+[1+2 \Phi] a^{2} d \vec{x} \cdot d \vec{x}
$$


Although we only need the first order deviations, the all-orders relations are,

$$
\begin{aligned}
\overline{\mathcal{T}}_{00}+\mathcal{T}_{00}=3 \sqrt{1+2 \Psi}\left[\frac{d}{d t}+3 H+\right. & \left.\frac{3 \dot{\Phi}}{1+2 \Phi}\right]\left[\frac{\left(H+\frac{\dot{\Phi}}{1+2 \Phi}\right)\left(\bar{\tau}+\delta \tau_{00}\right)}{(1+2 \Psi)^{\frac{3}{2}}}\right] \\
\overline{\mathcal{T}}_{i j}+\mathcal{T}_{i j}=-\frac{(1+2 \Phi) a^{2}}{\sqrt{1+2 \Psi}}\left[\frac{d}{d t}+3 H\right. & \left.+\frac{3 \dot{\Phi}}{1+2 \Phi}\right] \\
& \times \frac{1}{\sqrt{1+2 \Psi}}\left[\frac{d}{d t}+H+\frac{\dot{\Phi}}{1+2 \Phi}\right]\left[\frac{\bar{\tau}+\delta \tau_{00}}{1+2 \Psi}\right] .
\end{aligned}
$$

The fact that this simple result agrees with the direct computation is an excellent check on accuracy.

\section{Acknowledgements}

We are grateful for correspondence and conversations with C. Deffayet and S. Park. This work was partially supported by NSF grant PHY-1506513 and by the Institute for Fundamental Theory at the University of Florida.

\section{References}

[1] E. Aprile et al. [XENON Collaboration], Phys. Rev. Lett. 119, no. 18, 181301 (2017) doi:10.1103/PhysRevLett.119.181301 arXiv:1705.06655 [astro-ph.CO]].

[2] X. Cui et al. [PandaX-II Collaboration], Phys. Rev. Lett. 119, no. 18, 181302 (2017) doi:10.1103/PhysRevLett.119.181302 arXiv:1708.06917 [astro-ph.CO]].

[3] S. McGaugh, F. Lelli and J. Schombert, Phys. Rev. Lett. 117, no. 20, 201101 (2016) doi:10.1103/PhysRevLett.117.201101 arXiv:1609.05917 [astro-ph.GA]].

[4] F. Lelli, S. S. McGaugh, J. M. Schombert and M. S. Pawlowski, Astrophys. J. 836, no. 2, 152 (2017) doi:10.3847/1538-4357/836/2/152 arXiv:1610.08981 [astro-ph.GA]].

[5] M. Milgrom, Astrophys. J. 270, 365 (1983). doi:10.1086/161130

[6] M. Milgrom, Astrophys. J. 270, 371 (1983). doi:10.1086/161131

[7] M. Milgrom, Astrophys. J. 270, 384 (1983). doi:10.1086/161132 
[8] R. P. Woodard, Lect. Notes Phys. 720, 403 (2007) doi:10.1007/978-3540-71013-4_14 astro-ph/0601672.

[9] J. D. Bekenstein, Phys. Rev. D 70, 083509 (2004) Erratum: [Phys. Rev. D 71, 069901 (2005)] doi:10.1103/PhysRevD.70.083509, 10.1103/PhysRevD.71.069901 astro-ph/0403694].

[10] C. Skordis, D. F. Mota, P. G. Ferreira and C. Boehm, Phys. Rev. Lett. 96, 011301 (2006) doi:10.1103/PhysRevLett.96.011301 astro-ph/0505519].

[11] C. Skordis, Phys. Rev. D 74, $103513 \quad$ (2006) doi:10.1103/PhysRevD.74.103513 astro-ph/0511591].

[12] S. Dodelson and M. Liguori, Phys. Rev. Lett. 97, 231301 (2006) doi:10.1103/PhysRevLett.97.231301 [astro-ph/0608602].

[13] F. Bourliot, P. G. Ferreira, D. F. Mota and C. Skordis, Phys. Rev. D 75, 063508 (2007) doi:10.1103/PhysRevD.75.063508 [astro-ph/0611255].

[14] T. G. Zlosnik, P. G. Ferreira and G. D. Starkman, Phys. Rev. D 77, 084010 (2008) doi:10.1103/PhysRevD.77.084010 arXiv:0711.0520 [astro-ph]].

[15] C. R. Contaldi, T. Wiseman and B. Withers, Phys. Rev. D 78, 044034 (2008) doi:10.1103/PhysRevD.78.044034 [arXiv:0802.1215 [gr-qc]].

[16] P. C. C. Freire et al., Mon. Not. Roy. Astron. Soc. 423, 3328 (2012) doi:10.1111/j.1365-2966.2012.21253.x [arXiv:1205.1450 [astro-ph.GA]].

[17] R. Reyes, R. Mandelbaum, U. Seljak, T. Baldauf, J. E. Gunn, L. Lombriser and R. E. Smith, Nature 464, 256 (2010) doi:10.1038/nature08857 arXiv:1003.2185 [astro-ph.CO]].

[18] S. Dodelson, Int. J. Mod. Phys. D 20, 2749 (2011) doi:10.1142/S0218271811020561 [arXiv:1112.1320 [astro-ph.CO]].

[19] S. Boran, S. Desai, E. O. Kahya and R. P. Woodard, arXiv:1710.06168 [astro-ph.HE].

[20] S. Desai, E. O. Kahya and R. P. Woodard, Phys. Rev. D 77, 124041 (2008) doi:10.1103/PhysRevD.77.124041 [arXiv:0804.3804 [astro-ph]]. 
[21] T. G. Zlosnik, P. G. Ferreira and G. D. Starkman, Phys. Rev. D 75, 044017 (2007) doi:10.1103/PhysRevD.75.044017 astro-ph/0607411].

[22] R. H. Sanders, Phys. Rev. D 84, 084024 doi:10.1103/PhysRevD.84.084024 arXiv:1105.3910 [gr-qc]].

[23] L. Blanchet and S. Marsat, Phys. Rev. D 84, 044056 (2011) doi:10.1103/PhysRevD.84.044056 [arXiv:1107.5264 [gr-qc]].

[24] M. Milgrom, Phys. Rev. D 80, $123536 \quad$ (2009) doi:10.1103/PhysRevD.80.123536 arXiv:0912.0790 [gr-qc]].

[25] M. Milgrom, Phys. Rev. D 89, no. 2, 024027 (2014) doi:10.1103/PhysRevD.89.024027 arXiv:1308.5388 [gr-qc]].

[26] R. P. Woodard, Can. J. Phys. 93, no. 2, 242 (2015) doi:10.1139/cjp2014-0156 [arXiv:1403.6763 [astro-ph.CO]].

[27] C. L. Wang and R. P. Woodard, Phys. Rev. D 92, 084008 (2015) doi:10.1103/PhysRevD.92.084008 [arXiv:1508.01564 [gr-qc]].

[28] S. Park, T. Prokopec and R. P. Woodard, JHEP 1601, 074 (2016) doi:10.1007/JHEP01(2016)074 [arXiv:1510.03352 [gr-qc]].

[29] M. B. Frb and E. Verdaguer, JCAP 1603, no. 03, 015 (2016) doi:10.1088/1475-7516/2016/03/015 [arXiv:1601.03561 [hep-th]].

[30] C. Deffayet, G. Esposito-Farese and R. P. Woodard, Phys. Rev. D 84, 124054 (2011) doi:10.1103/PhysRevD.84.124054 arXiv:1106.4984 [grqc]].

[31] C. Deffayet, G. Esposito-Farese and R. P. Woodard, Phys. Rev. D 90, no. 6, 064038 (2014) Addendum: [Phys. Rev. D 90, no. 8, 089901 (2014)] doi:10.1103/PhysRevD.90.089901, 10.1103/PhysRevD.90.064038 [arXiv:1405.0393 [astro-ph.CO]].

[32] M. Kim, M. H. Rahat, M. Sayeb, L. Tan, R. P. Woodard and B. Xu, Phys. Rev. D 94, no. 10, 104009 (2016) doi:10.1103/PhysRevD.94.104009 [arXiv:1608.07858 [gr-qc]]. 
[33] P. A. R. Ade et al. [Planck Collaboration], Astron. Astrophys. 594, A13 (2016) doi:10.1051/0004-6361/201525830 arXiv:1502.01589 [astroph.CO]].

[34] A. G. Riess et al., Astrophys. J. 826, no. 1, 56 (2016) doi:10.3847/0004637X/826/1/56 [arXiv:1604.01424 [astro-ph.CO]].

[35] S. Nojiri and S. D. Odintsov, Phys. Lett. B 659, 821 (2008) doi:10.1016/j.physletb.2007.12.001 arXiv:0708.0924 [hep-th]].

[36] S. Deser and R. P. Woodard, JCAP 1311, 036 (2013) doi:10.1088/14757516/2013/11/036 [arXiv:1307.6639 [astro-ph.CO]].

[37] R. P. Woodard, Found. Phys. 44, 213 (2014) doi:10.1007/s10701-0149780-6 [arXiv:1401.0254 [astro-ph.CO]].

[38] M. E. Soussa and R. P. Woodard, Class. Quant. Grav. 20, 2737 (2003) doi:10.1088/0264-9381/20/13/321 astro-ph/0302030].

[39] S. Deser and R. P. Woodard, Phys. Rev. Lett. 99, 111301 (2007) doi:10.1103/PhysRevLett.99.111301 [arXiv:0706.2151 [astro-ph]].

[40] I. Arraut, Int. J. Mod. Phys. D 23, 1450008 (2014) doi:10.1142/S0218271814500084 [arXiv:1310.0675 [gr-qc]].

[41] C. Deffayet and R. P. Woodard, JCAP 0908, 023 (2009) doi:10.1088/1475-7516/2009/08/023 [arXiv:0904.0961 [gr-qc]].

[42] S. Park and S. Dodelson, Phys. Rev. D 87, no. 2, 024003 (2013) doi:10.1103/PhysRevD.87.024003 arXiv:1209.0836 [astro-ph.CO]].

[43] S. Dodelson and S. Park, Phys. Rev. D 90, 043535 (2014) doi:10.1103/PhysRevD.90.043535 [arXiv:1310.4329 [astro-ph.CO]].

[44] H. Nersisyan, A. F. Cid and L. Amendola, JCAP 1704, no. 04, 046 (2017) doi:10.1088/1475-7516/2017/04/046 arXiv:1701.00434 [astroph.CO]].

[45] S. Park, arXiv:1711.08759 [gr-qc].

[46] J. D. Bekenstein and E. Sagi, Phys. Rev. D 77, 103512 (2008) doi:10.1103/PhysRevD.77.103512 [arXiv:0802.1526 [astro-ph]]. 
[47] M. Milgrom, Astrophys. J. 698, 1630 (2009) doi:10.1088/0004637X/698/2/1630 [arXiv:0810.4065 [astro-ph]]. 